\title{
Total Number of Synapses in the Adult Human Neocortex
}

Thai Nguyen

University of South Florida

Advisors:

Arcadii Grinshpan, Mathematics and Statistics

Andrei Chugunov, Fortis College: Medical Sciences

Problem Suggested By: Andrei Chugunov

Follow this and additional works at: https://digitalcommons.usf.edu/ujmm

Part of the Mathematics Commons

UJMM is an open access journal, free to authors and readers, and relies on your support:

Donate Now

\section{Recommended Citation}

Nguyen, Thai (2010) "Total Number of Synapses in the Adult Human Neocortex," Undergraduate Journal of Mathematical Modeling: One + Two: Vol. 3: Iss. 1, Article 14.

DOI: http://dx.doi.org/10.5038/2326-3652.3.1.26

Available at: https://digitalcommons.usf.edu/ujmm/vol3/iss1/26 


\title{
Total Number of Synapses in the Adult Human Neocortex
}

\begin{abstract}
The brain is composed of glial cells and neurons where synapses form connections between neurons and other cells. Since synapses are very small, so either a light or electron microscope is required to see them. Unlike other mammals, synapses in the human brain deteriorate rapidly upon death making them difficult to study. This project constructs a simple model for the number of synapses in the human neocortex by age and sex based on the amount of neurons. This hypothetical model can also be used to study the impact of Alzheimer's disease and other forms of dementia that are marked by a decreased number of synaptic connections.
\end{abstract}

\section{Keywords}

Gende, Synaptic Density, Aging

Creative Commons License

(c) (i) (8)

This work is licensed under a Creative Commons Attribution-Noncommercial-Share Alike 4.0 License.

\section{Erratum}

This article was previously called Article 26. 


\section{TABLE OF CONTENTS}

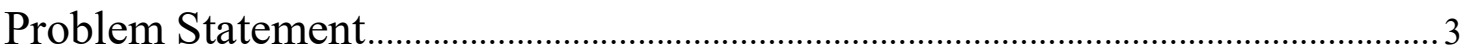

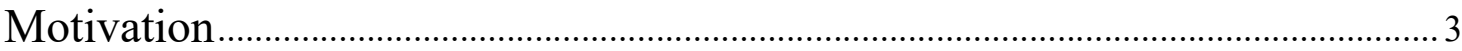

Mathematical Description and Solution Approach ................................................. 4

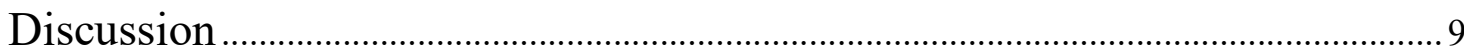

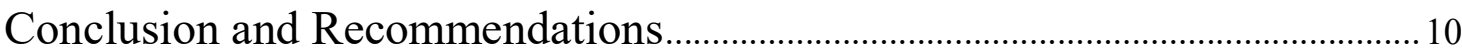

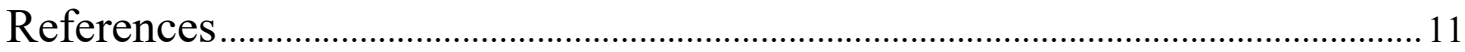

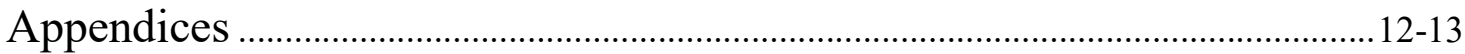




\section{PROBLEM STATEMENT}

The brain is composed of two types of cells: glial cells and neurons. Glial cells provide structural and metabolic support for the brain. Neurons are excitable cells which chemically transmit electrical signals through connections called synapses. There is a consensus that there are roughly about 100 billion neurons total in the human brain. Each of these neurons can have up to 15,000 connections with other neurons via synapses (Brotherson).

Even with this knowledge about the number of neurons and synapses, there is not a commonly accepted model for the number of synapses in human neocortex as a function of time. A sample model can be generated using the studies by Pakkenberg, on the degradation of neocortical neurons over time by estimating the average number of synapses each neuron can form (Pakkenberg and Gundersen). Combining these facts, we can construct a hypothetical model for the number of synapses found in the neocortex of the human brain.

\section{MOTIVATION}

The brain is the organ which controls all other organs and even though that brain accounts for only $5 \%$ of a human's total body weight, it uses $20 \%$ of the body's oxygen while at rest (Gusnard and Raichle). Although the brain has been actively researched for years, many mysteries still remain concerning both its development and operation.

In particular, the human neocortex is composed of six layers and controls the senses, muscle movement, spatial reasoning, language and conscious thought. For this reason, a large portion of neurology research is focused on understanding how the neocortex functions. Anatomically the neocortex sends and receives messages through the synapses of neurons. 
Studying and collecting data on synapses may give rise to the treatment of various mental disorders, improve drug development, and give rise to new technologies based on the workings of the brain.

\section{MATHEMATICAL DESCRIPTION AND SOLUTION APPROACH}

We build our model for the number synapses by first estimating the number of neurons in the neocortex as a function of time. Next we estimate the number of synapses per neuron over time. Putting these two models together will yield a model for the number of synapses based on a person's age. We also account for the recent studies which have shown that gender greatly affects the quantity of both neurons (Pakkenberg and Gundersen) and synapses (AlonsoNanclares, Gonzalez-Soriano and Rodriguez) in the cerebral cortex.

\section{$\underline{\text { NEURONS }}$}

There is still some controversy on the amount of neurons lost due to aging. Several studies, (Terry, De Teresa and Hansen) and (Haug, Kuhl and Mecke), have claimed that the total neuron population does not decrease as we age, but rather neurons may become smaller and fall into different size classes. However, it is held by (Pakkenberg, Pelvig and Marnera) that neurons are lost as we age. In particular Pakkenberg estimates a $9.8 \%$ loss of neurons from the age of 20 to 90 or around $85,000 /$ day.

We shall assume the model given by Pakkenberg and Gunderson for the rate of neuron loss in the male neocortex over time as

$$
N_{\text {Male }}(t)=10^{9} e^{3.201-0.00145 t}
$$


and the female neocortex over time as

$$
N_{\text {Female }}(t)=10^{9} e^{3.151-0.00145 t}
$$

Their model accounts for both gender and age. Figure 1 shows the relationship between (1), (2), and the number of neocortical neurons from 94 sampled brains (Pakkenberg and Gundersen).

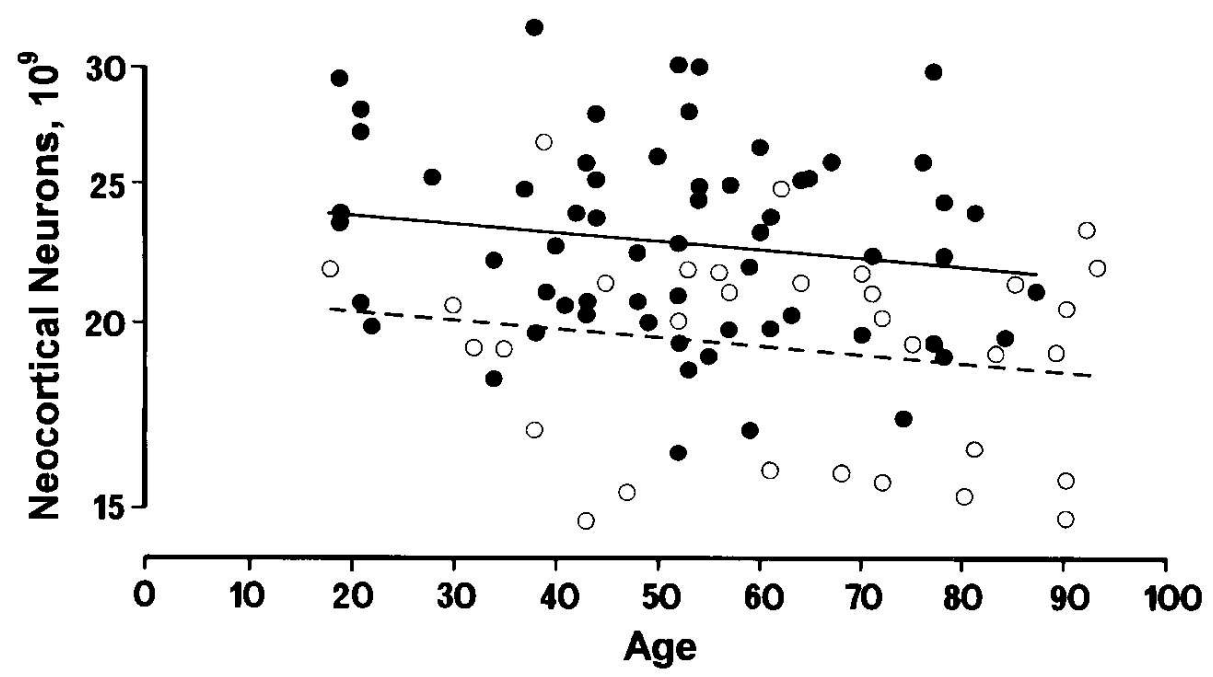

Figure 1: The number of neocortical neurons in the human brain (Pakkenberg and Gunderson, 1997). Total neuron number as a function of age (linear scale) in males (solid circles) and females (open circles). The two regression lines are indicated for males (solid line) and females (dashed line).

\section{SYNAPSES}

We now turn our attention to the number of synapses per neuron. Recently it has been shown that a person's sex contributes to their synaptic density:

"As a consequence, the number of synapses in each layer was greater in men than in women, and, thus, in this particular region of the neocortex the general connectivity in men appears to be more extensive than in women. Accordingly, gender appears to 
influence synaptic connectivity, and this phenomenon is regulated independent of other cytoarchitectonic features." (Alonso-Nanclares, Gonzalez-Soriano and Rodriguez)

In particular, Alonso-Nanclares et al. found that certain biopsied neocortical tissue in males contained approximately 57\% more synapses per neuron (see Table 2). Moreover, the differences in synaptic densities were present in all six cortical layers (Appendix - Figure 5). Unlike other studies, which obtained their samples postmortem, Alonso-Nanclares et al. obtained their samples from patients undergoing surgical treatment for epilepsy. This means that the number of neurons and synapses may not accurately reflect the true numbers found in a normal human neocortex; however the discrepancies in synaptic densities between the genders should be unaffected by the illness.

\begin{tabular}{|ccc|}
\hline Sex & $\begin{array}{c}\text { Number of synapses } \\
\left(\times \mathbf{1 0}^{\mathbf{8}} / \mathbf{m m}^{3}\right)\end{array}$ & $\begin{array}{c}\text { Number of neurons } \\
\left(/ \mathbf{m m}^{3}\right)\end{array}$ \\
\hline Women & $7.17 \pm 3.29$ & $27,589 \pm 16,854$ \\
\hline Men & $10.61 \pm 4.97$ & $25,924 \pm 15,110$ \\
\hline
\end{tabular}

Table 2: Number of cortical synapses and neurons by sex sampled from a biopsies of patients undergoing surgical treatment for epilepsy. (Alonso-Nanclares, Gonzalez-Soriano and Rodriguez)

It should be noted that the higher neural density in women observed by Alonso-Nanclares et al. does not immediately contradict the results of Pakkenberg and Gunderson. The average human male's cerebral cortex is approximately $650.10 \mathrm{~cm}^{3}$ compared to $573.54 \mathrm{~cm}^{3}$ in the female (Carne, Vogrin and Litewka) so a higher density does not directly translate to a higher overall count. Moreover Pakkenberg and Gunderson investigated the Brodmann area 21 of the cerebral cortex while Alonso-Nanclares et al. focused on the Brodmann area 22. However, the increased synaptic density in males cannot be explained in the same way. Considering the 
increased cerebral volume in males, the increased synaptic density translates to a further increase in overall synaptic count.

Finally we consider the effects of aging on the number of cortical synapses. Throughout normal brain development, new synapses will form while others are lost. The effects of age on synaptic density are best summarized in Volume 14 of the review Cerebral Cortex:

"More studies are needed to determine if there is a loss of synapses from cerebral cortex of human and nonhuman primates in normal aging, and whether such loss is specific for certain cortical areas or global. But based on the available evidence, the tentative conclusion is that, except perhaps for the frontal cortex, normal aging brings only a small decrease in the number of synapses." (Neurodegenerative and Age-Related Changes in Structure and Function of Cerebral Cortex)

\section{Synaptic Density in Normal Aging}

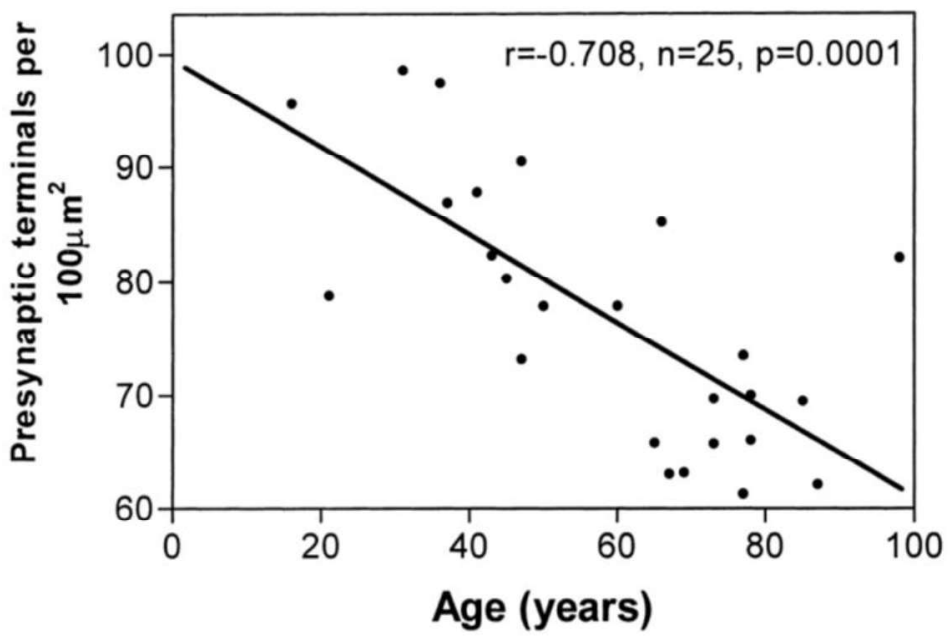

Figure 3: The density of presynaptic terminals measured by confocal microscopy of vibratome sections of superior prefrontal gyrus reacted with antisynaptophysin plotted against the patient's age (Masliah, Mallory and Hansen). 
Masliah et al. and Gibson both observed an inverse relationship between presynaptic terminals and age in the frontal cortex (Fig. 3). In particular Masliah indicated a 20\% decrease in frontal cortex synapses between individuals older than 65 years versus individuals 60 year or younger. Likewise Gibson observed a 20\% loss in synapses between the ages of 16 and 90 .

We model the $20 \%$ frontal cortex synaptic loss due to aging as

$$
F(t)=F_{16}\left(1-\frac{2}{10}\left(\frac{t-16}{90-16}\right)\right)=F_{16}(1.0432-0.0027 t)
$$

where $F(t)$ is number of synapses per neurons in the frontal cortex at year $t$ and $F_{16}$ is the number of synapses per neuron at 16 . Table 5 in the Appendix says that $F(21.8) \approx 7540 \frac{\mathrm{syn}}{\mathrm{neu}}$ which allows us to compute that $F_{16} \approx 7660 \frac{\text { syn }}{n e u}$ and gives

$$
F(t) \approx 7991.32-20.7 t
$$

Using table 5 and considering no loss in the other cortical regions we can compute the global synapse to neuron ratio for the male neocortex as a function of time:

$$
\begin{aligned}
R(t) & =\frac{69}{590}(4360)+\frac{149}{590}(6330)+\frac{133}{590}(8950)+\frac{239}{590}(7991.32-20.7 t) \\
& =7355.62-8.39 t .
\end{aligned}
$$

Multiplying (1) by (5) yields a model for the total number of synapses in the male neocortex over time:

$$
S_{\text {male }}(t)=\left(1.806 \times 10^{14}-2.06 \times 10^{11} t\right) e^{-0.00145 t} .
$$

From Alonso-Nanclares et al., we estimate that female neocortex has a synapse to neuron ratio of $0.64 R(t)$ which combined with (2) gives

$$
S_{\text {female }}(t)=\left(1.091 \times 10^{14}-1.245 \times 10^{11} t\right) e^{-0.00145 t} .
$$


The total number of synapses for both the male and female neocortex is plotted in Figure 4.

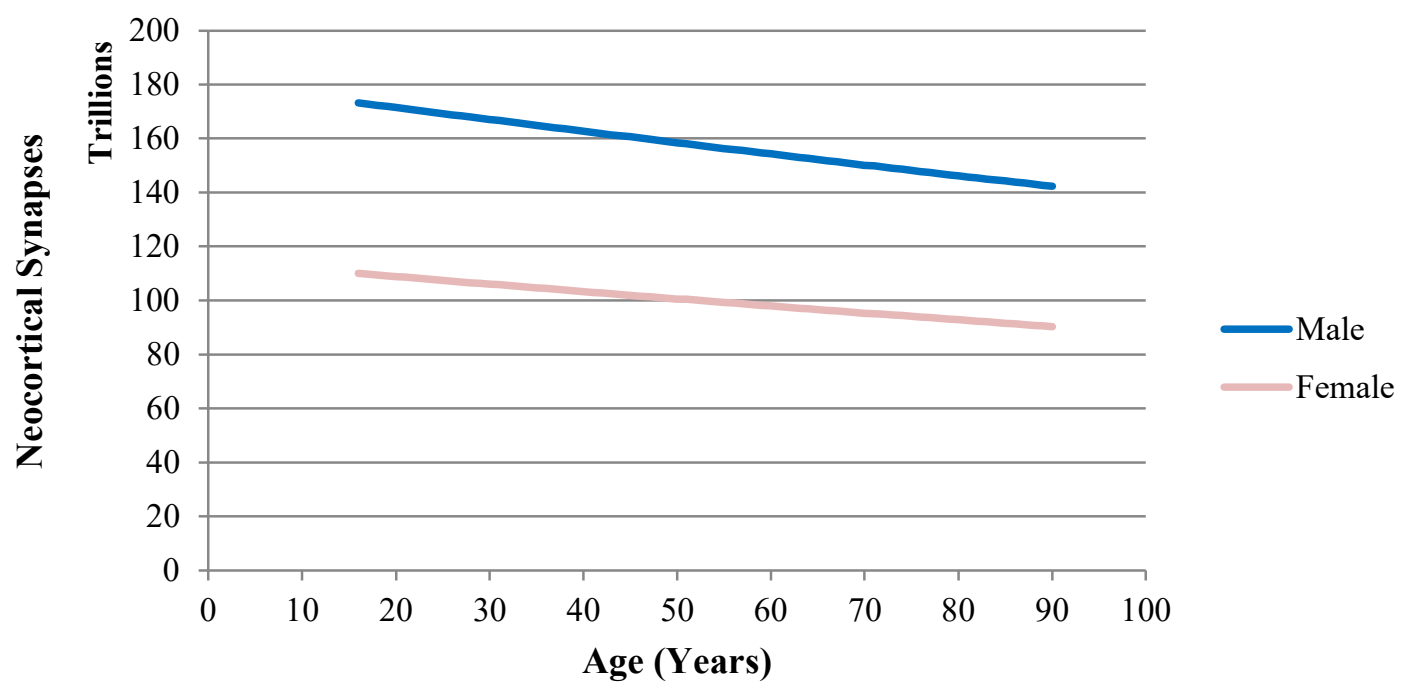

Figure 4: The estimated number of neocortical synapses in the male (blue) and female (pink) human brain based on age.

\section{DISCUSSION}

From Pakkenberg and Gundersen we estimate the number of neurons for each sex over time to be (1) and (2) and number of respective synapses to be (6) and (7). However our models are reliant on the precision of these previous studies and the assumption of uniform densities for both neurons and synapses among the neocortex.

The assumption of uniform neocortex density is inconsistent with the findings of Pakkenberg and Gundersen versus that of Alonso-Nanclares et al. Pakkenberg showed a higher neuron count in the male Brodmann area 21 while Alonso-Nanclares listed a higher neuron count in the female Brodmann area 22. The most likely cause for their findings is non-uniformity in neuron density for various regions of the human neocortex. As new studies are done, our model can be revised to reflect more accurate density variations. 
Finally, it should be mentioned that there is not a direct correlation between neuron and synapse count and performance. Moreover these findings do not suggest a more complex neural network in the male brain. For simplicity, the total number of neocortical synapses from (6) and (7) do not specific how many symmetrical/asymmetric or neuron-to-neuron synapses for each gender. More studies are required for a more complete picture.

\section{CONCLUSION AND RECOMMENDATIONS}

Recent research has shown that both gender and age nontrivially affect the number of neurons and synapses in the human neocortex. Technological limitations presently prevent the collection of an accurate synaptic count in a living human, so models for both neurons and synapses are meaningful tools in modern research.

Based on the data and models of Pakkenberg and Gundersen, Masliah et al., and AlonsoNanclares et al., we were able to generate a graphical presentation for the total number of synapses in the adult human male and female neocortex as a function of age. This model indicates that the human male neocortex contains a higher count of both neurons and synapses than the female. Additionally, the number of neocortical neurons and synapses of both sexes seem to decline a part of the normal aging process.

With increasing technology, one day every synapse in a human brain could be counted. Recently Stanford University of School of Medicine was able to use array tomography to produce an extremely detailed image of the neural connections in a mouse (Goldman). Although this method will not work on living animals, it may still be the next step in unraveling the mystery of nature's human supercomputer, the brain. 


\section{REFERENCES}

Alonso-Nanclares, L, et al. "Gender Differences in Human Cortical Synaptic Density." Proceedings of the National Academy of Sciences of the United States of America. 2008. 14615-14619.

Brotherson, Sean. "Understanding Brain Development in Young Children, FS-609." n.d. NDSU Extension Service. April 2009. <http://www.ag.ndsu.edu/pubs/yf/famsci/fs609w.htm>.

Carne, R P, et al. "Cerebral cortex: An MRI-based study of volume and variance with age and sex." Journal of Clinical Neuroscience 13.1 (2006): 60-72.

Gibson, P H. "EM study of the number of cortical synapses in the brains of aging people and people with Alzheimer-type dementias." Acta neuropathologica 62 (1983): 127-133.

Goldman, Bruce. New imaging method developed at Stanford reveals stunning details of brain connections. 17 November 2010. $<$ http://med.stanford.edu/ism/2010/november/neuronimaging.html>.

Gusnard, D. A. and M. E. Raichle. "Searching for a baseline: Functional imaging and the resting human brain." Nature Reviews Neuroscience 2 (2001).

Haug, H, et al. "The significance of morphometric procedures in the investigation of age changes in cytoarchitectonic structures of human brain." J. Hirnforsch. 25 (1984): 353-374.

Masliah, E, et al. "Quantitative synaptic alterations in the human neocortex during normal aging." Neurology 43 (1993): 192-197.

"Neurodegenerative and Age-Related Changes in Structure and Function of Cerebral Cortex." Cerebral Cortex. Ed. Alan Peteres and John H Morrison. Vol. 14. New York: Kluwer Academic / Plenum Publishers, 1999. 49-87.

Pakkenberg, B. and H. J. G. Gundersen. "Neocortical neuron number in humans: Effect of sex and age." Journal of Comparative Neurology 384.2 (1997): 312-320.

Pakkenberg, B., et al. "Aging and the human neocortex." Experimental Gerontology 38 (2003): 95-98.

Tang, Yong, et al. "Total Regional an Global Number of Synapses in the Human Brain Neocortex." Synapse 41 (2001): 258-273.

Terry, R and R Katzman. "Life span and synapses: Will there be a primary senile dementia?" Neurobiology of Aging 22 (2001): 347-348.

Terry, R, R De Teresa and L Hansen. "Neocortical cell counts in normal human adult aging." Ann. Neurol. 21 (1987): 530-539. 


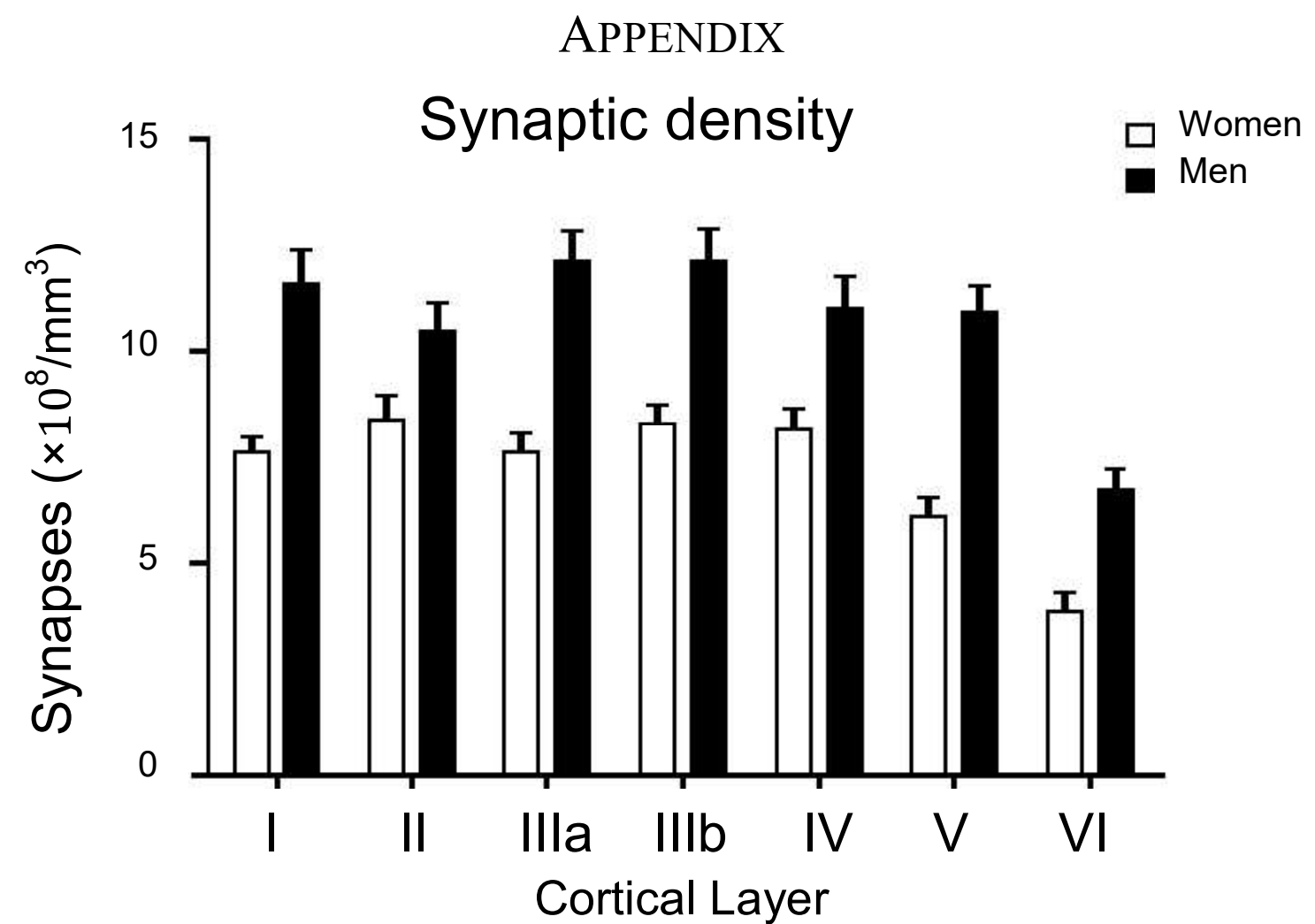

Figure 5: Graph showing a comparison of cortical synaptic density between men and women in each cortical layer. (Alonso-Nanclares, Gonzalez-Soriano and Rodriguez)

Synaptic Density in Normal Aging

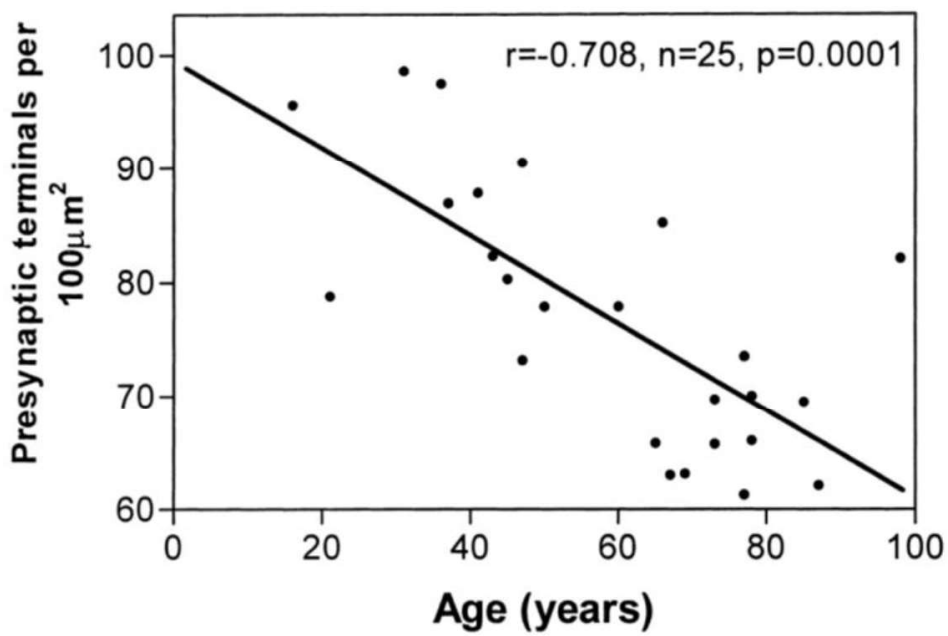

Figure 6: The density of presynaptic terminals measured by confocal microscopy of vibratome sections of superior prefrontal gyrus reacted with antisynaptophysin plotted against the patient's age (Masliah, Mallory and Hansen). 


\begin{tabular}{|c|c|c|c|c|c|c|}
\hline No. & $\begin{array}{c}\text { Age } \\
\text { (years) }\end{array}$ & $\begin{array}{c}\text { Region } \\
\text { (lobe) }\end{array}$ & $\begin{array}{c}\text { Volume } \\
\left(\mathrm{cm}^{3}\right)\end{array}$ & $\begin{array}{c}\text { Synapses } \\
\left(10^{12}\right)\end{array}$ & $\begin{array}{c}\text { Neurons } \\
\left(10^{9}\right)\end{array}$ & $\begin{array}{c}\text { Syn/Neu } \\
\left(10^{3}\right)\end{array}$ \\
\hline \multirow{4}{*}{1} & \multirow{4}{*}{19} & Occipital & 67 & 13.6 & 4.28 & 3.17 \\
\hline & & Parietal & 155 & 47.8 & 6.97 & 6.87 \\
\hline & & Temporal & 152 & 56.5 & 6.57 & 8.60 \\
\hline & & Frontal & 247 & 67.2 & 7.50 & 8.96 \\
\hline \multirow{4}{*}{2} & \multirow{4}{*}{19} & Occipital & 57 & 15.1 & 5.21 & 2.89 \\
\hline & & Parietal & 151 & 40.2 & 6.68 & 6.02 \\
\hline & & Temporal & 129 & 34.7 & 4.18 & 8.28 \\
\hline & & Frontal & 195 & 40.8 & 7.89 & 5.18 \\
\hline \multirow{4}{*}{3} & \multirow{4}{*}{21} & Occipital & 71 & 11.0 & 3.29 & 3.34 \\
\hline & & Parietal & 152 & 43.9 & 5.01 & 8.78 \\
\hline & & Temporal & 156 & 42.3 & 4.32 & 9.81 \\
\hline & & Frontal & 278 & 77.1 & 7.42 & 10.4 \\
\hline \multirow{4}{*}{4} & \multirow{4}{*}{22} & Occipital & 60 & 15.3 & 3.10 & 4.95 \\
\hline & & Parietal & 135 & 21.4 & 5.73 & 3.73 \\
\hline & & Temporal & 117 & 44.5 & 3.73 & 11.9 \\
\hline & & Frontal & 233 & 57.6 & 8.26 & 6.97 \\
\hline \multirow{4}{*}{5} & \multirow{4}{*}{28} & Occipital & 90 & 54.9 & 7.37 & 7.44 \\
\hline & & Parietal & 151 & 54.0 & 8.64 & 6.25 \\
\hline & & Temporal & 110 & 31.9 & 5.21 & 6.13 \\
\hline & & Frontal & 243 & 51.8 & 8.38 & 6.18 \\
\hline \multirow{5}{*}{ Mean } & \multirow{5}{*}{21.8} & Occipital & 69 & 22.0 & 4.56 & 4.36 \\
\hline & & Parietal & 149 & 41.5 & 6.61 & 6.33 \\
\hline & & Temporal & 133 & 42.0 & 4.8 & 8.95 \\
\hline & & Frontal & 239 & 58.9 & 7.89 & 7.54 \\
\hline & & Total & 590 & 164 & 23.9 & 6.93 \\
\hline
\end{tabular}

Table 7: Estimates relating to total number of synapses in five human male brains. (Tang, Nyengaard and De Groot) 